\title{
Leaky-wave-induced disks around Be stars: a pulsational analysis on their formation
}

\author{
Melanie Godart ${ }^{1}$, Hiromoto Shibahashi ${ }^{1}$ and Marc-Antoine Dupret ${ }^{2}$ \\ ${ }^{1}$ Dept. of Astronomy, The University of Tokyo, Japan \\ email: melanie.godart@gmail.com \\ ${ }^{2}$ Dept. of Astrophysics, Geophysics and Oceanography, University of Liège, Belgium
}

\begin{abstract}
Be stars are B-type stars near the main sequence which undergo episodic mass loss events detected by emission lines, whose line shape and intensity vary with a timescale of the order of decades. Spectroscopic observations show a large rotation velocity such that one of the prevailing scenarios for the formation of the equatorial disk consists in an increasing equatorial rotation velocity to the break-up limit where gravity is challenged by the centrifugal force. We investigate here a new scenario recently suggested by Ishimatsu \& Shibahashi (2013), in which the transport of angular momentum through the photosphere would be achieved by leaky waves, keeping the rotation velocity still below the break-up limit.
\end{abstract}

Keywords. stars: emission-line, Be, oscillations, rotation

\section{Leaky-wave-induced disks - scenario}

The emission lines in the spectrum of Be stars are now widely accepted to be due to episodic mass-loss from the equatorial region of the star, occurring quasi-periodically and forming a cool circumstellar disk (e.g. Porter \& Rivinius 2003). One of the prevailing scenarios for the formation of the equatorial disk consists in an increasing equatorial rotation velocity to the break-up limit where gravity is challenged by the centrifugal force. Whether Be stars rotate at the critical break-up limit or not (e.g. Ekström et al. 2012; Granada et al. 2012) remains unclear although their high rotation places Be stars in the center of discussions about angular momentum (AM) transport. Non-radial oscillations have then been investigated as another mechanism to transport the AM and increase the rotation up to the break-up velocity (e.g. Neiner et al. 2013). Recently, Ishimatsu \& Shibahashi (2013); Shibahashi (2014) suggested a new scenario in which the transport of AM through the photosphere would be achieved by leaky waves, keeping the rotation velocity still below the break-up limit. In this scenario, g-modes (excited by the $\kappa$-mechanism operating in the iron opacity bump) redistribute the AM but the wave leakage through the photosphere prevents a large deposition of AM at the stellar surface keeping a lower surface velocity. The waves are not fully reflected at the stellar surface and loose therefore their standing wave characterisctic. A reflection occurs when the frequency of the mode is larger than a critical frequency. This latter one increases with the rotation velocity and the reflective boundary may no longer occur for a high rotation rate, allowing the wave leakage and the transport of AM to the circumstellar envelope to form a disk. The wave loses then its energy and the oscillations are damped. Episodic mass loss would occur in a timescale of the order of the mode lifetime. 


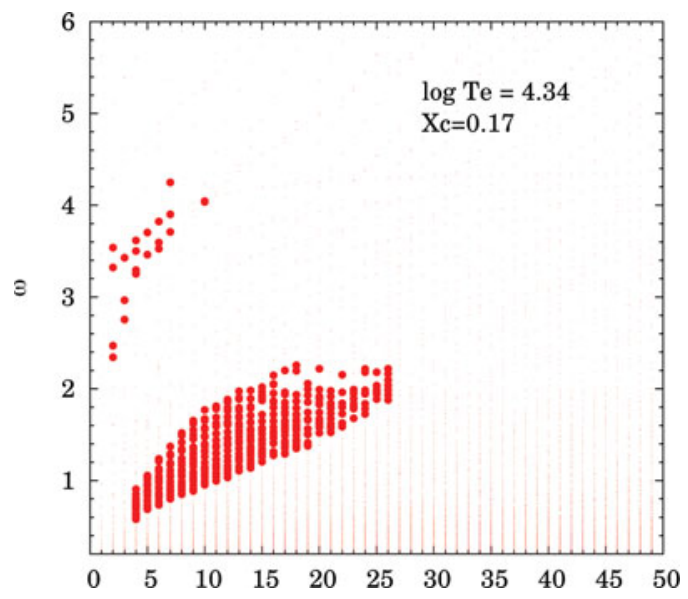

Figure 1. Dimensionless angular frequency range for excited modes computed for $l=1$ to 50 in a $10 M_{\odot}$ model with $X_{\mathrm{c}}=0.17$. Red dots stand for the stable modes while solid red circles stand for the unstable modes.

\section{Stellar and pulsational models}

Stellar evolution models were computed with CLES (Scuflaire et al. 2008). We adopted the AGS05 metal mixture (Asplund et al. 2005), the corresponding OP opacity tables (Badnell et al. 2005), $X=0.070$ and $Z=0.014$ and a convective core overshooting of 0.1 . Non-adiabatic computations have been performed with MAD (Dupret 2002), in which the traditional approximation for rotation has been implemented (Bouabid et al. 2013) to perform modes for $1 \leqslant l \leqslant 4$. The efficiency of wave leakage (given by the integral over the surface of the wave kinetic energy) should increase with the rotation as the critical frequency increases. We derive an increase by $75 \%$ of the wave leakage between $\Omega / \Omega_{\mathrm{c}}=0.37$ and $\Omega / \Omega_{\mathrm{c}}=0.55$ showing, indeed, an increasing efficiency of the leaking effect with rotation. These results are however preliminary and further investigation need to be carry on. The lack of observational evidence of non-radial oscillations in all Be stars is not invalidating the scenario. Indeed, high degree $l$ oscillation modes are expected to be excited in the Be star mass range. Figure 1 shows the range of dimensionless angular frequencies for g-modes $(l=1$ to 50$)$ in a $10 \mathrm{M}_{\odot}$ model (without rotation) near the end of the main sequence. $l=4$ to 26 modes are found unstable. The lifetimes of such modes are in good agreement with the quasi-periodic Be phenomenom (9.45 year for a mode of $l=15, \omega=1.25, P=12 \mathrm{~h})$.

\section{Acknowledgements}

This research has been funded by the Japanese Society for Promotion of Science (JSPS).

\section{References}

Asplund, M., Grevesse, N., \& Sauval, A. J. 2005, in T. G. Barnes, III \& F. N. Bash (eds.), Cosmic Abundances as Records of Stellar Evolution and Nucleosynthesis, Vol. 336 of Astronomical Society of the Pacific Conference Series, p. 25

Badnell, N. R., Bautista, M. A., Butler, K., et al. 2005, MNRAS 360, 458

Bouabid, M.-P., Dupret, M.-A., Salmon, S., et al. 2013, MNRAS 429, 2500

Dupret, M.-A. 2002, Non-radial non-adiabatic oscillations of near main sequence variable stars, Vol. 71 
Ekström, S., Georgy, C., Granada, A., Wyttenbach, A., \& Meynet, G. 2012, in R. CapuzzoDolcetta, M. Limongi, \& A. Tornambè (eds.), Advances in Computational Astrophysics: Methods, Tools, and Outcome, Vol. 453 of Astronomical Society of the Pacific Conference Series, p. 353

Granada, A., Ekström, S., Georgy, C., et al. 2012, in A. C. Carciofi \& T. Rivinius (eds.), Circumstellar Dynamics at High Resolution, Vol. 464 of Astronomical Society of the Pacific Conference Series, p. 117

Ishimatsu, H. \& Shibahashi, H. 2013, in H. Shibahashi \& A. E. Lynas-Gray (eds.), Astronomical Society of the Pacific Conference Series, Vol. 479 of Astronomical Society of the Pacific Conference Series, p. 325

Neiner, C., Mathis, S., Saio, H., \& Lee, U. 2013, in H. Shibahashi \& A. E. Lynas-Gray (eds.), Astronomical Society of the Pacific Conference Series, Vol. 479 of Astronomical Society of the Pacific Conference Series, p. 319

Porter, J. M. \& Rivinius, T. 2003, PASP 115, 1153

Scuflaire, R., Théado, S., Montalbán, J., et al. 2008, ApESSS 316, 83

Shibahashi, H. 2014, in J. A. Guzik, W. J. Chaplin, G. Handler, \& A. Pigulski (eds.), IAU Symposium, Vol. 301 of IAU Symposium, pp 173-176

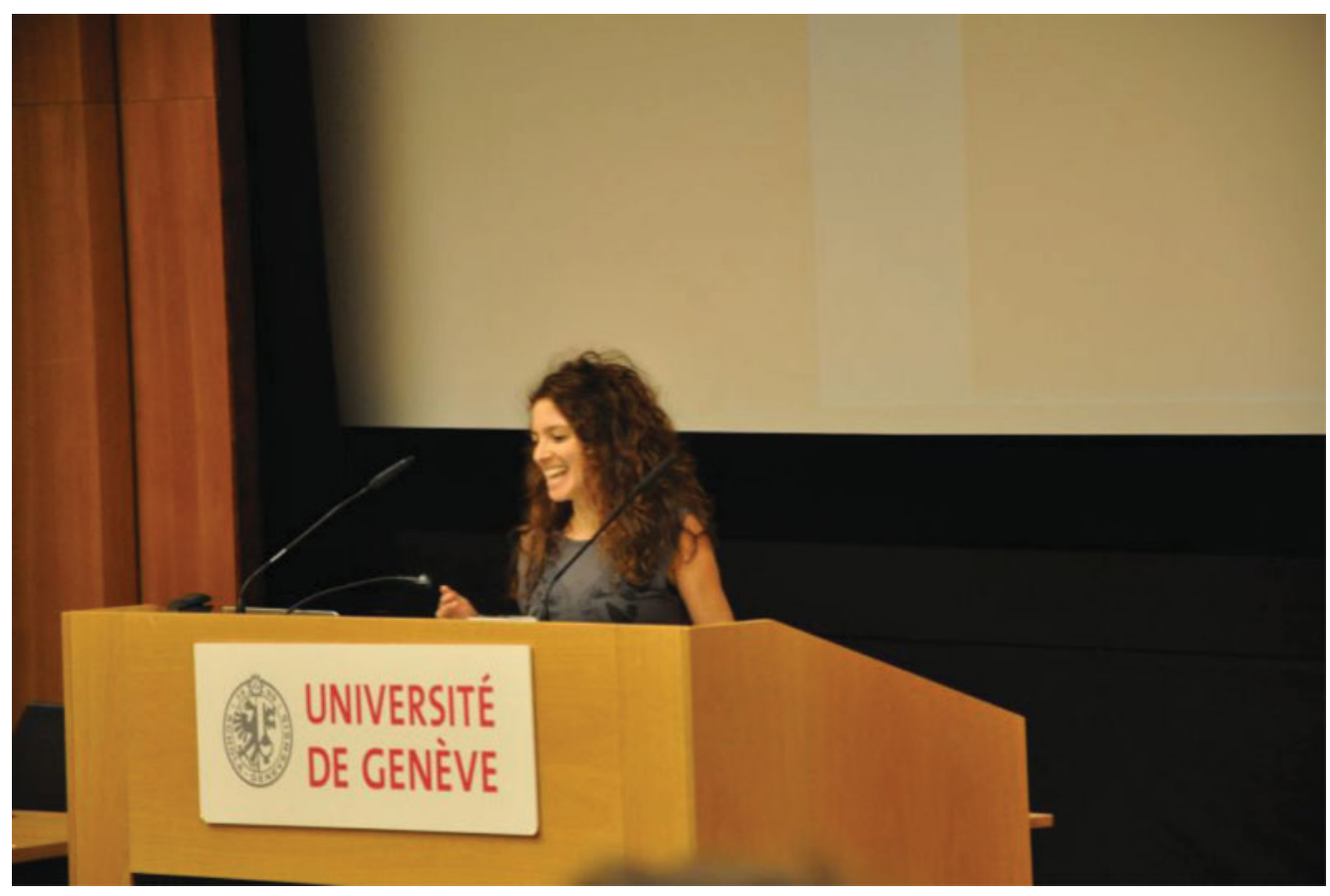

Mélanie Godart 